\title{
Desempenho silvicultural e seleção precoce de clones de híbridos de eucalipto
}

\author{
Rafael Beltrame(1), Dilson Antônio Bisognin ${ }^{(1)}$, Bruno Dufau Mattos ${ }^{(2)}$, Alberto Cargnelutti Filho(1), \\ Clovis Roberto Haselein(1), Darci Alberto Gatto(2) e Gleison Augusto dos Santos ${ }^{(3)}$
}

\begin{abstract}
(1)Universidade Federal de Santa Maria, Avenida Roraima, no 1.000, Caixa Postal 5.096, CEP 97105-900 Santa Maria, RS. E-mail: browbeltrame@yahoo.com.br, dilsonb@smail.ufsm.br, cargnelutti@pq.cnpq.br, clovis.haselein@smail.ufsm.br ${ }^{(2)}$ Universidade Federal de Pelotas, Rua Conde Porto Alegre, no 793, CEP 96010-290 Pelotas, RS. E-mail: brunodufaumattos@yahoo.com.br, darcigatto@yahoo.com ${ }^{(3)}$ CMPC Celulose Riograndense, Rua São Geraldo, no 1.680, CEP 92500-000 Guaíba, RS, E-mail: gaugusto@cmpcrs.com.br
\end{abstract}

Resumo - O objetivo deste trabalho foi avaliar a variabilidade genética e o desempenho silvicultural de clones de híbridos interespecíficos de Eucalyptus urophylla, E. globulus, E. maidenii, E. saligna, E. grandis, E. pellita, E. resinifera, E. kirtoniana e $E$. dunnii, e determinar a viabilidade da seleção precoce em selecionar clones superiores. Os diâmetros do tronco à altura do peito aos três (DAP3) e sete (DAP7) anos de idade e a altura total das árvores aos sete anos de idade foram mensurados. Utilizou-se o delineamento experimental de blocos ao acaso, com 138 clones, 10 repetições e seis plantas por parcela. Os dados foram submetidos à análise de variância e agrupados pelo método das k-médias, tendo-se realizado a comparação de médias e determinado a correlação de Pearson (r) entre as variáveis avaliadas. A validação do agrupamento foi realizada por meio de análise de variância e do teste de Tukey. Os clones de híbridos interespecíficos de Eucalyptus apresentaram variabilidade genética. Foram estabelecidos cinco grupos de clones com base no desempenho silvicultural (DAP3, DAP7 e altura). O DAP3 é altamente correlacionado ao DAP7 e à altura aos sete anos de idade. A seleção com base no DAP3 pode ser utilizada para identificar clones superiores de Eucalyptus com grande vigor de crescimento.

Termos para indexação: Eucalyptus, agrupamento de clones, ganho de seleção, melhoramento genético, seleção indireta, silvicultura clonal.

\section{Silvicultural performance and early selection of clones from eucalyptus hybrids}

\begin{abstract}
The objective of this work was to evaluate the genetic variability and silvicultural performance of clones from interspecific hybrids of Eucalyptus urophylla, E. globulus, E. maidenii, E. saligna, E. grandis, E. pellita, E. resinifera, E. kirtoniana and E. dunnii, and to determine the feasibility of early selection in selecting superior clones. The stem diameter at breast height at three (DAP3) and seven years (DAP7) and the total tree height at seven years were evaluated. A randomized complete block design, with 138 clones, ten replicates and six plants per plot was used. Data were subjected to analysis of variance and clustered by the k-means method; the means were compared, and the Pearson correlation (r) was determined between variables. Clustering validation was performed using analysis of variance and Tukey's test. Clones of interspecific Eucalyptus hybrids showed genetic variability. Five groups of clones were established based on their silvicultural performance (DAP3, DAP7 and height). The DAP3 is highly correlated to DAP7 and height at seven years. The selection based on DAP3 can be used to identify superior clones of Eucalyptus with good growth vigor.
\end{abstract}

Index terms: Eucalyptus, clone groups, selection gain, plant breeding, indirect selection, clonal silviculture.

\section{Introdução}

O gênero Eucalyptus é amplamente utilizado no Brasil para a produção de madeira com diversas finalidades, em razão de seu rápido crescimento, boa adaptação ecológica e alta qualidade da madeira. Todavia, a maioria das florestas desse gênero é manejada para a produção de pequenos fustes, para o uso em indústrias de celulose ou energia. Essas florestas foram implantadas e manejadas com pouca preocupação com a qualidade da madeira e, atualmente, o aproveitamento desse material é prejudicado quando se requer usos de maior valor agregado (Ponce, 1995). As raras florestas destinadas para a produção de toras para serraria não foram implantadas com material genético selecionado e, apesar da boa forma e do diâmetro, a madeira apresenta alta variação quanto à massa específica e às propriedades mecânicas. 
A utilização de clones foi um avanço muito importante, que possibilitou aumentar a produção e uniformizar a qualidade da madeira, ao gerar florestas de alta qualidade para satisfazer a demanda industrial de matéria prima (Berger et al., 2002). A seleção de híbridos com boas características silviculturais e tecnológicas é o objetivo primordial dos programas de melhoramento genético de Eucalyptus no Brasil. Nesses programas, os híbridos são avaliados quanto aos diferentes caracteres e ambientes, antes da seleção final, multiplicação e recomendação para a exploração comercial (Nunes et al., 2002).

Para os programas de melhoramento genético de espécies florestais, o tempo é um fator crítico, pois a exploração envolve ciclos longos, demorados e onerosos. O eucalipto faz parte desse grupo e pode apresentar idades de rotação entre 25 e 30 anos, em regiões de clima temperado, e de aproximadamente 10 anos em climas tropicais (Pereira et al., 1997). Assim, alternativas que visam à diminuição do tempo para que se complete uma geração de melhoramento são necessárias, e é vantajoso aos programas de melhoramento praticar seleção de genótipos superiores com base nos primeiros anos de produção. Uma das alternativas encontradas é o emprego da seleção precoce (Massaro et al., 2010). A seleção precoce visa identificar caracteres das árvores em idade juvenil correlacionados àqueles de interesse econômico em árvores em idades mais avançadas, isto é, predizer, em idade juvenil, o desempenho de um indivíduo adulto, diminuindo, assim, o tempo para se completar um ciclo de seleção (Gonçalves et al., 1998).

A eficiência da seleção precoce pode ser avaliada por meio das expectativas de ganhos indiretos, observados ao corte, de acordo com a seleção em idades juvenis (Marques Junior, 1995). A possibilidade de prever os ganhos quanto a diferentes critérios de seleção é considerada uma das maiores contribuições da genética quantitativa para o melhoramento de plantas (De Paula et al., 2002). Portanto, o desempenho silvicultural de uma floresta poderia ser previsto pelas curvas de projeção do crescimento em diâmetro, altura e volume, o que simplificaria o planejamento (Berger et al., 2002). Isso é possível pelo fato de as variáveis diâmetro, altura e volume apresentarem correlações significativas entre si, em diferentes idades, o que viabiliza a eficiência na seleção precoce do material genético (Massaro et al., 2010).
O objetivo deste trabalho foi avaliar a variabilidade genética e o desempenho silvicultural de clones de híbridos interespecíficos de Eucalyptus urophylla, E. globulus, E. maidenii, E. saligna, E. grandis, E. pellita, E. resinifera, E. kirtoniana e E. dunnii, e determinar a viabilidade da seleção precoce em selecionar clones superiores.

\section{Material e Métodos}

O material genético utilizado proveio de um teste clonal de híbridos interespecíficos de Eucalyptus urophylla S.T. Blake, E. globulus Labill., E. maidenii F. Muell., E. saligna Smith, E. grandis W. Mill ex Maiden, E. pellita F. Muell., E. resinifera Smith in J. White, E. kirtoniana F. Muell. e E. dunnii Maiden. O experimento foi realizado no Município de Tapes, RS (30³4'34"S, 51³0'50"W, a $103 \mathrm{~m}$ de altitude), com clima subtropical úmido, em área pertencente à empresa CMPC Celulose Riograndense. O povoamento foi implantado em agosto de 2003, com espaçamento inicial de 3,0x3,0 m, com 138 clones oriundos de hibridações interespecíficas. Utilizou-se o delineamento experimental de blocos ao acaso, com dez repetições de seis plantas (60 árvores por clone), no total de 8.280 árvores, em área de aproximadamente 7,3 ha.

Foram mensurados: o diâmetro do tronco à altura do peito, aos três (DAP3, cm) e aos sete anos (DAP7, cm), e a altura total das árvores aos sete anos (m). As mensurações dos diâmetros foram efetuadas com fita métrica, e as de altura com o Vertex III (Haglöf, Sweden).

A análise de variância para os caracteres DAP3, DAP7 e altura foi realizada, tendo-se considerado o delineamento de blocos completos ao acaso. Com os dados obtidos, foi feito o diagrama de dispersão, para visualizar as relações entre os caracteres. Foi, então, determinada a matriz de coeficientes de correlação linear de Pearson (r) entre DAP3, DAP7 e altura, e verificada a significância dos coeficientes, por meio do teste $\mathrm{t}$ de Tukey, a $5 \%$ de probabilidade, com 136 graus de liberdade.

Para separar os 138 clones, de acordo com os caracteres, estabeleceram-se, a priori, cinco grupos, e agruparam-se os clones por meio do método das k-médias (Barroso \& Artes, 2003; Mingoti, 2005). Para validar o agrupamento, foi feita a análise de variância univariada. 
O ganho genético (indireto) de seleção, para os três caracteres avaliados, foi calculado por GS $=$ MCS $\mathrm{MCO}$, em que: GS é o ganho genético de seleção (\%); MCS é a média do grupo de clones selecionados; e MCO é a média de todos os clones avaliados.

As análises estatísticas foram realizadas pelo programa Genes (Cruz, 2006).

\section{Resultados e Discussão}

O coeficiente de variação experimental (CVe) foi maior do que o de variação amostral para todos os caracteres (Tabela 1), o que evidencia maior variabilidade entre as parcelas, em comparação à variabilidade entre as plantas dentro das parcelas. Os elevados valores de acurácia seletiva $(\mathrm{AS} \geq 0,93)$ e de herdabilidade $\left(h^{2} \geq 0,87\right)$ indicam precisão experimental bastante alta, de acordo com os limites estabelecidos em Resende \& Duarte (2007), Cargnelutti Filho \& Storck (2009) e Cargnelutti Filho et al. (2009).

As estatísticas AS e $\mathrm{h}^{2}$ foram consideradas mais adequadas para a avaliação da precisão experimental do que o coeficiente de variação $(\mathrm{CV})$. Os maiores escores de $\mathrm{AS}$ e de $\mathrm{h}^{2}$ estão associados à maior variabilidade genética e menor variância residual, o que indica experimentos mais precisos (Cargnelutti Filho \& Storck, 2007, 2009). Também constatou-se efeito significativo de clones $(p \leq 0,05)$, quanto aos caracteres DAP3, DAP7 e altura, o que evidencia a existência de variabilidade genética, fundamental para a viabilidade de seleção de materiais genéticos superiores.

Tabela 1. Análise de variância do experimento, com média, coeficiente de variação experimental (CVe), coeficiente de variação amostral (CVa), acurácia seletiva e herdabilidade, para diâmetro à altura do peito aos três (DAP3) e aos sete (DAP7) anos de idade, e altura de planta de 138 clones de Eucalyptus spp.

\begin{tabular}{|c|c|c|c|c|}
\hline \multirow[t]{3}{*}{ Fontes de variação } & \multirow[t]{3}{*}{ GL } & \multicolumn{3}{|c|}{ Quadrado médio } \\
\hline & & DAP3 & DAP7 & \multirow{2}{*}{$\begin{array}{c}\text { Altura } \\
\text { (m) }\end{array}$} \\
\hline & & \multicolumn{2}{|c|}{---------------(cm)-------------- } & \\
\hline Bloco & 9 & $136,49 *$ & $298,14^{*}$ & $846,36^{*}$ \\
\hline Clone & 137 & $186,81 *$ & $1.298,50^{*}$ & $1.305,53^{*}$ \\
\hline Erro experimental & 1.233 & $24,21 *$ & $56,84^{*}$ & $96,44 *$ \\
\hline Erro amostral & 6.036 & 2,40 & 11,23 & 11,94 \\
\hline Média & - & 12,97 & 18,46 & 24,89 \\
\hline $\mathrm{CVe}(\%)$ & - & 37,91 & 40,82 & 39,45 \\
\hline CVa (\%) & - & 11,94 & 18,15 & 13,88 \\
\hline Acurácia seletiva & - & 0,93 & 0,98 & 0,96 \\
\hline Herdabilidade $^{(1)}$ & - & 0,87 & 0,96 & 0,92 \\
\hline
\end{tabular}

(1) Média de clones*Significativo pelo teste F, a $5 \%$ de probabilidade.
No presente estudo, ficou evidente a forte correlação linear de Pearson entre as variáveis analisadas, independentemente das diferenças genéticas existentes entre os clones de híbridos interespecíficos (Figura 1). Os elevados coeficientes de correlação confirmam a associação linear positiva entre os caracteres avaliados. Esses resultados mostram que a seleção indireta de clones com maior DAP7 e maior altura pode ser realizada precocemente, considerando-se somente DAP3.

Alguns estudos comprovam que a viabilidade do uso da seleção precoce pode ser utilizada para diminuir os ciclos e aumentar o ganho genético de seleção. Em espécies de Eucalyptus, a seleção precoce pode ser realizada considerando-se caracteres de crescimento, como diâmetro, altura e volume, em razão da grande variabilidade genética para esses caracteres (Tolfo, 2003). A seleção precoce pode ser realizada em diferentes idades, e tem sido praticada eficientemente em diversas espécies como o Eucalyptus camaldulensis Dehnh (Pereira et al.,1997); Pinus (Xiang et al., 2003; Weng et al., 2007); Hevea brasiliensis (Willd. ex A. Juss.) Müll. Arg. (Gonçalves et al., 1998) e Euterpe oleracea Mart. (Bovi et al., 1990).

A análise de variância mostrou, quanto aos três caracteres avaliados, que a variabilidade entre grupos é superior à variabilidade entre clones dentro de grupo - com base no desempenho silvicultural dos 138 clones (Tabela 2) -, e que há cinco grupos, de acordo com agrupamento realizado por meio do método das k-médias (Barroso \& Artes, 2003; Mingoti, 2005).

O agrupamento facilita a identificação de clones com desempenho silvicultural superior ou com maior vigor de crescimento. Cabe ressaltar que, antes da recomendação para o cultivo, são necessárias a avaliação e a análise de outros caracteres, tais como massa específica, biomassa do lenho, interações fenotípicas e genotípicas, índices de tensões de crescimento e qualidade da madeira (De Paula et al., 2002; Nunes et al., 2002), já que estas são importantes para a seleção de clones com alto potencial genético. No entanto, estas avaliações podem ser efetuadas exclusivamente nos clones selecionados quanto ao maior vigor de crescimento, identificados no DAP3.

Além de haver diferenças significativas entre grupos de clones, o teste de Tukey mostrou que as médias de grupos diferiram entre si (Tabela 3), o que indica a 
ocorrência de eficiência no processo de agrupamento. As diferenças significativas entre grupos, quanto aos três caracteres avaliados, não influenciou os altos valores de correlação encontrados entre os caracteres avaliados (Figura 1).

Com base nos resultados, podem-se propor estratégias de seleção do material genético de acordo com o objetivo do melhoramento e da demanda comercial. O grupo 1 é composto por $18 \%$ do total de clones, e este grupo, em associação com o grupo 2, soma $42 \%$ dos clones em avaliação. Se a principal estratégia de seleção for o alto vigor de crescimento, poderiam ser selecionados apenas os 25 clones do grupo 1. No entanto, se a estratégia for identificar clones que combinam vigor de crescimento com outras características, seria importante selecionar os clones dos grupos 1 e 2, ou seja, 58 clones do total de 138 clones em avaliação. Portanto, aos três anos de idade já poderiam ser eliminados $58 \%$ dos clones em avaliação, o que possibilitaria avaliar clones de outras progênies e, com isso, maximizar o ganho genético de seleção com os mesmos recursos financeiros e humanos disponíveis.

A seleção dos clones do grupo 1 (índice de seleção de $18 \%$ ) ou dos grupos 1 e 2 (índice de seleção de $42 \%$ ) resultaria em ganho indireto maior, quanto às variáveis altura e DAP7, do que quanto ao DAP3 (Tabela 4). No entanto, mesmo que fossem selecionados 58 dos 138 clones, ainda seria obtido um ganho indireto de seleção de 10,94\% para DAP3, ou seja, um ganho anual superior a 3\%. O maior ganho indireto de seleção foi obtido para DAP7.
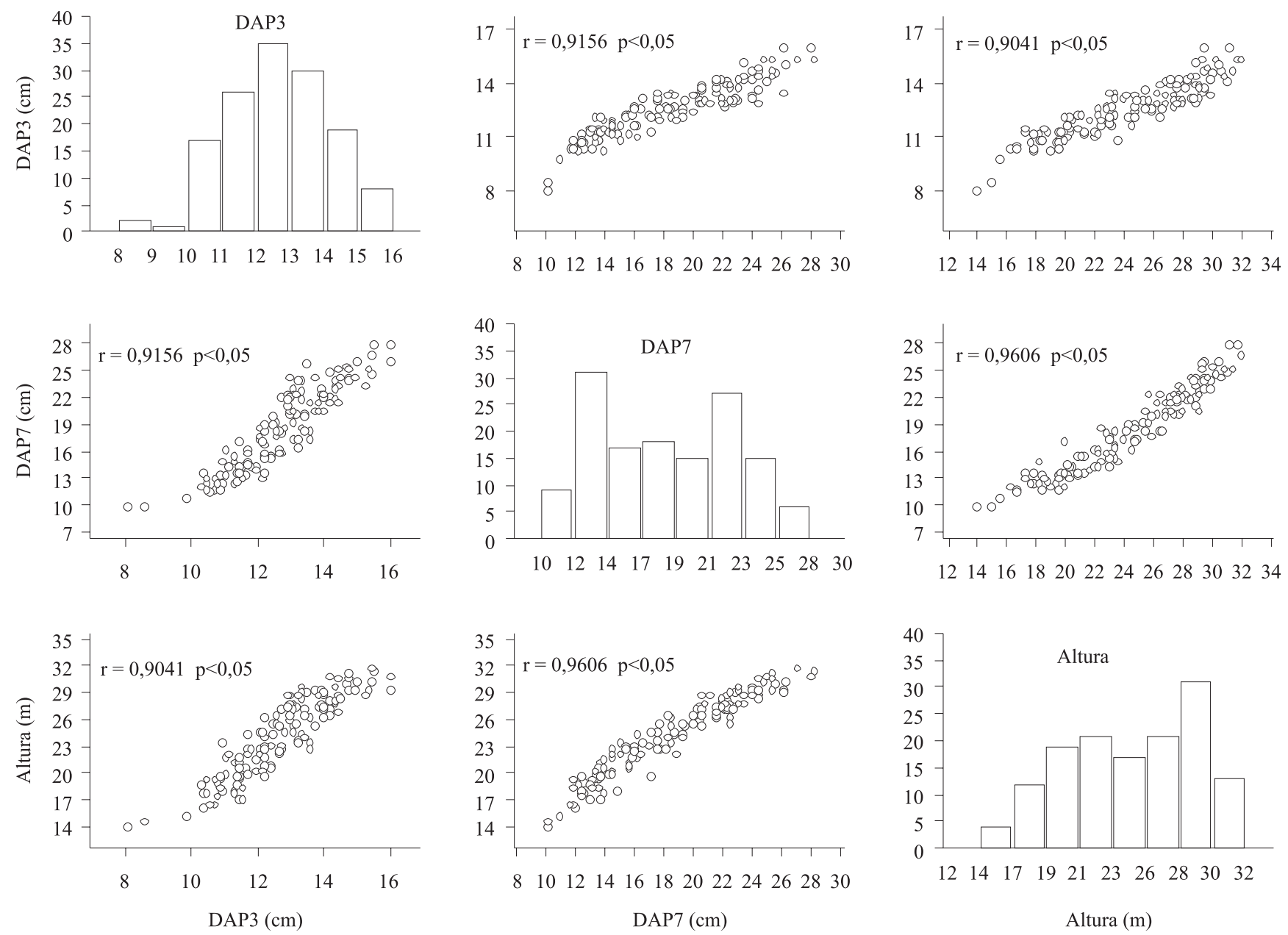

Figura 1. Distribuição de frequências (na diagonal descendente da esquerda para a direita) e gráficos de dispersão do diâmetro à altura do peito aos três (DAP3) e aos sete (DAP7) anos de idade, e da altura de plantas, de 138 clones de Eucalyptus spp. O r refere-se ao coeficiente de correlação de Pearson (r) entre os caracteres, e o p à significância estatística da correlação. 
O alto coeficiente de correlação linear $(0,92)$ entre DAP3 e DAP7, as diferenças significativas entre os grupos de clones e o alto ganho genético de seleção, quanto aos três caracteres avaliados, indicam que a seleção com base no DAP3 é uma estratégia viável para a identificação precoce de clones de Eucalyptus

Tabela 2. Análise de variância do agrupamento, com média e coeficiente de variação, para os diâmetros à altura do peito aos três (DAP3) e aos sete (DAP7) anos de idade, e altura de planta de 138 clones de híbridos interespecíficos de Eucalyptus spp.

\begin{tabular}{lcccc}
\hline Causas de & GL & \multicolumn{3}{c}{ Quadrado médio } \\
\cline { 3 - 5 } variação & & DAP3 & DAP7 & $\begin{array}{c}\text { Altura } \\
(\mathrm{m})\end{array}$ \\
\hline Grupo & 4 & $61,87^{*}$ & $674,76^{*}$ & $608,12^{*}$ \\
Erro & 133 & 0,42 & 1,16 & 1,36 \\
\hline Média & - & 12,89 & 18,18 & 24,61 \\
CV $(\%)$ & - & 5,03 & 5,94 & 4,74 \\
\hline
\end{tabular}

*Significativo pelo teste $\mathrm{F}$, a $5 \%$ de probabilidade.

Tabela 3. Médias do diâmetro à altura do peito aos três (DAP3) e aos sete (DAP7) anos de idade, e altura da planta dos grupos formados de híbridos interespecíficos de Eucalyptus $\operatorname{spp}^{(1)}$.

\begin{tabular}{lcccc}
\hline Grupos $^{(2)}$ & № de clones & DAP3 & $\begin{array}{c}\text { DAP7 } \\
\text {---------(cm)------- }\end{array}$ & $\begin{array}{c}\text { Altura } \\
(\mathrm{m})\end{array}$ \\
\hline 1 & 25 & $14,84 \mathrm{a}$ & $24,66 \mathrm{a}$ & $30,23 \mathrm{a}$ \\
2 & 33 & $13,77 \mathrm{~b}$ & $21,43 \mathrm{~b}$ & $27,85 \mathrm{~b}$ \\
3 & 24 & $12,90 \mathrm{c}$ & $17,88 \mathrm{c}$ & $24,77 \mathrm{c}$ \\
4 & 28 & $12,09 \mathrm{~d}$ & $14,72 \mathrm{~d}$ & $21,90 \mathrm{~d}$ \\
5 & 28 & $10,91 \mathrm{e}$ & $12,32 \mathrm{e}$ & $18,38 \mathrm{e}$ \\
\hline
\end{tabular}

${ }^{(1)}$ Médias seguidas de letras iguais não diferem entre si, pelo teste $t$ de Tukey, a 5\% de probabilidade. ${ }^{(2)}$ Método das k-médias.

Tabela 4. Médias dos grupos selecionados de clones (MCS), médias originais dos clones (MCO) e ganho genético (GS) e percentual (GS) indireto na seleção de clones do grupo 1 e dos grupos $1 \mathrm{e} 2$, quanto ao diâmetro à altura do peito aos três (DAP3) e aos sete (DAP7) anos de idade, e à altura de planta de clones de híbridos interespecíficos de Eucalyptus spp.

\begin{tabular}{lcccc}
\hline Caractere & MCS & MCO & GS & GS (\%) \\
\hline & \multicolumn{4}{c}{ Seleção de clones do grupo 1 } \\
DAP3 $(\mathrm{cm})$ & 14,84 & 12,89 & 1,95 & 15,12 \\
DAP7 $(\mathrm{cm})$ & 24,66 & 18,18 & 6,48 & 35,64 \\
Altura $(\mathrm{m})$ & 30,23 & 24,61 & 5,62 & 22,83 \\
\hline \multicolumn{5}{c}{ Seleção de clones dos grupos 1 e 2 } \\
DAP3 (cm) & 14,30 & 12,89 & 1,41 & 10,94 \\
DAP7 (cm) & 23,04 & 18,18 & 4,86 & 26,73 \\
Altura (m) & 29,04 & 24,61 & 4,43 & 18,00 \\
\hline
\end{tabular}

com alto vigor de crescimento. Se o objetivo do melhoramento for a seleção de clones quanto a outros caracteres, principalmente de baixa herdabilidade, um maior número de clones deve ser mantido no programa, a fim de possibilitar alto ganho genético para estes caracteres; além disso, pode-se aumentar a variabilidade genética na população, o que também proporcionaria maior ganho genético por seleção.

Resultados semelhantes foram encontrados por De Paula et al. (2002), que indicaram a existência de variabilidade genética entre as famílias de Eucalyptus camaldulensis quanto ao DAP e à altura, e sugeriram a possibilidade de ganhos genéticos pela seleção dos melhores genótipos. Os resultados observados no presente estudo corroboram os obtidos por Kalil Filho et al. (1983), Santos et al. (1990), Silva (1990) e Cornacchia (1994), em estudos com outras espécies florestais.

Observa-se, em trabalhos com Eucalyptus no Brasil, que, apesar de a idade de rotação ser menor do que em outros países, a seleção precoce também temse mostrado eficiente (Rezende et al., 1994; Pereira et al.,1997; Tolfo, 2003).

\section{Conclusões}

1. Clones de híbridos interespecíficos de Eucalyptus spp. apresentam variabilidade genética suficiente para serem separados em grupos quanto aos diâmetros do tronco à altura do peito (DAP), aos três e aos sete anos, e quanto à altura total das árvores aos sete anos de idade.

2. O diâmetro do tronco à altura do peito (DAP) aos três anos é altamente correlacionado ao DAP e à altura total das árvores aos sete anos de idade.

3. O DAP aos três e aos sete anos de idade, e altura de planta aos sete anos de idade apresentam ganhos genéticos satisfatórios para seleção de clones.

4. A seleção precoce aos três anos após o plantio pode ser empregada para identificar clones de híbridos de Eucalyptus com bom vigor de crescimento.

\section{Agradecimentos}

Ao Conselho Nacional de Desenvolvimento Científico e Tecnológico, pelas bolsas de estudo e de produtividade em pesquisa; e à empresa CMPC Celulose Riograndense, pelo apoio e assistência técnica para a realização do trabalho. 


\section{Referências}

BARROSO, L.P.; ARTES, R. Análise multivariada. Lavras: UFLA, 2003. 151p.

BERGER, R.; SCHNEIDER, P.R.; FINGER, C.A.G.; HASEILEN, C.R. Efeito do espaçamento e da adubação no crescimento de um clone de Eucalyptus saligna Smith. Ciência Florestal, v.12, p.75-87, 2002.

BOVI, M.L.A.; GODOY JÚNIOR, G.; SPIERING, S.H.; CAMARGO, S.B. de. Correlações fenotípicas entre caracteres avaliados nos estádios juvenil e adulto de açaizeiros. Bragantia, v.49, p.321-334, 1990.

CARGNELUTTI FILHO, A.; STORCK, L. Estatísticas de avaliação da precisão experimental em ensaios de cultivares de milho. Pesquisa Agropecuária Brasileira, v.42, p.17-24, 2007.

CARGNELUTTI FILHO, A.; STORCK, L. Medidas do grau de precisão experimental em ensaios de competição de cultivares de milho. Pesquisa Agropecuária Brasileira, v.44, p.111-117, 2009.

CARGNELUTTI FILHO, A.; STORCK, L.; RIBEIRO, N.D. Medidas da precisão experimental em ensaios com genótipos de feijão e de soja. Pesquisa Agropecuária Brasileira, v.44, p.1225-1231, 2009.

CORNACCHIA, G. Variabilidade genética em procedência de $P$. caribaea var. hondurensis Barr. \& Golf., P. oocarpa Schiede e $P$. tecunumanii (Schw.) Eguiluz \& Perry na região oeste do Estado da Bahia. 1994. 155p. Dissertação (Mestrado) Universidade Federal de Viçosa, Viçosa.

CRUZ, C.D. Programa genes: estatística experimental e matrizes. Viçosa: UFV, 2006. 285p.

DE PAULA, R.C.; PIRES, I.E.; BORGES, R. de C.G.; CRUZ, C.D. Predição de ganhos genéticos em melhoramento florestal. Pesquisa Agropecuária Brasileira, v.37, p.159-165, 2002.

GONÇALVES, P. de S.; BORTOLETTO, N.; FONSECA, F. da.; BATAGLIA, O.C.; ORTOLANI, A.A. Early selection for growth vigor in rubber tree genotypes in northwestern São Paulo state (Brazil). Genetics and Molecular Biology, v.21, p.620-630, 1998.

KALIL FILHO, A.N.; PIRES, C.L.S.; FONTES, M.A. Análise do comportamento e estimação de parâmetros genéticos em progênies de Pinus elliottii Engelm. var. elliottii na região de Itararé (SP). Silvicultura, v.8, p.325-326, 1983.

MARQUES JÚNIOR, O.G. Estimativas dos parâmetros genéticos e fenotípicos e avaliação da eficiência da seleção precoce em Eucalyptus cloeziana F. Muell. 1995. 69p. Dissertação (Mestrado) - Universidade Federal de Lavras, Lavras.
MASSARO, R.A.M.; BONINE, C.A.V.; SCARPITANI, E.A.; DE PAULA, R.C. Viabilidade de aplicação da seleção precoce em testes clonais de Eucalyptus spp. Ciência Florestal, v.20, p.597-609, 2010.

MINGOTI, S.A. Análise de dados através de métodos de estatística multivariada. Belo Horizonte: UFMG, 2005. 297p.

NUNES, B.H.S.; REZENDE, G.D.S.P.; RAMALHO, M.A.P.; SANTOS, J.B. Implicações da interação genótipo $\mathrm{x}$ ambientes na seleção de clones de eucalipto. Cerne, v.8, p.49-58, 2002.

PEREIRA, A.B.; MARQUES JUNIOR, O.G.; RAMALHO, M.A.P.; ALTHOFF, P. Eficiência da seleção precoce em famílias de meios-irmãos de Eucalyptus camaldulensis Dehnh., avaliadas na região noroeste do Estado de Minas Gerais. Cerne, v.3, p.67-81, 1997.

PONCE, R.H. Madeira serrada de eucalipto: desafios e perspectivas. In: SEMINÁRIO INTERNACIONAL DE UTILIZAÇÃO DA MADEIRA DE EUCALIPTO PARA SERRARIA, 1995. São Paulo. Anais. Piracicaba: IPEF/IPT, 1995. p.50-58.

RESENDE, M.D.V. de; DUARTE, J.B. Precisão e controle de qualidade em experimentos de avaliação de cultivares. Pesquisa Agropecuária Tropical, v.37, p.182-194, 2007.

REZENDE, G.D.S.P.; BERTOLUCCI, F. de L.G.; RAMALHO, M.A.P. Eficiência da seleção precoce na recomendação de clones de eucalipto avaliados no norte do Espírito Santo e sul da Bahia. Cerne, v.1, p.45-50, 1994.

SANTOS, P.E.T. dos; GARCIA, C.H.; MORI, E.S.; MORAES, M.L.T. de. Potencial para programas de melhoramento, estimativas de parâmetros genéticos e interação progênies $\mathrm{x}$ locais em populações de Eucalyptus urophylla S. T. Blake. IPEF, n.43/44, p.11-19, 1990.

SILVA, J.F. Variabilidade genética em progênies de Eucalyptus camaldulensis Dehnh. e sua interação com espaçamentos. 1990. 110p. Dissertação (Mestrado) - Universidade Federal de Viçosa, Viçosa.

TOLFO, A.L.T. Estudos da viabilidade de aplicação da seleção precoce em testes clonais de Eucalyptus spp. e qualidade da madeira para polpa celulósica. 2003. 50p. Dissertação (Mestrado) - Universidade Estadual Paulista, Jaboticabal.

WENG, Y.H.; TOSH, K.J.; PARK, Y.S.; FULLARTON, M.S. Age-related trends in genetic parameters for jack pine and their implications for early selection. Silvae Genetica, v.56, p.242-252, 2007.

XIANG, B.; LI, B.; ISIK, F. Time trend of genetic parameters in growth traits of Pinus taeda L. Silvae Genetica, v.52, p.114-121, 2003.

Recebido em 13 de dezembro de 2011 e aprovado em 1o de junho de 2012

Pesq. agropec. bras., Brasília, v.47, n.6, p.791-796, jun. 2012 